\title{
Near-Infrared Laser Direct Writing of conductive patterns at the surface of Carbon nanotubes polymer nanocomposites
}

\author{
Gerges El Haber, ${ }^{1,2,5}$ Laurent Noel, ${ }^{1,2}$ Ching-Fu Lin,,${ }^{1,2,3,4}$ Simon Gree, ${ }^{1,2}$ Loic Vidal, ${ }^{1,2}$ Hsiao-Wen \\ Zan, ${ }^{3,4}$ Nelly Hobeika, ${ }^{5}$ Olivier Lhost, ${ }^{6}$ Yves Trolez, ${ }^{6}$ Olivier Soppera ${ }^{1,2, *}$ \\ ${ }^{1}$ Université de Haute-Alsace, CNRS, IS2M UMR 7361, F-68100 Mulhouse, France \\ ${ }^{2}$ Université de Strasbourg, France \\ ${ }^{3}$ Department of Photonics and Institute of Electro-Optical Engineering, College of Electrical and \\ Computer Engineering, National Yang Ming Chiao Tung University, Hsinchu, 300, Taiwan, ROC \\ ${ }^{4}$ Department of Photonics and Institute of Electro-Optical Engineering, College of Electrical and \\ Computer Engineering, National Chiao Tung University, Hsinchu, 300, Taiwan, ROC \\ ${ }^{5}$ Lebanese University, Faculty of Engineering Branch 2, Roumieh, Metn, Mount-Lebanon, Lebanon. \\ ${ }^{6}$ TotalEnergies OneTech Belgium, 7181 Feluy, Belgium \\ * corresponding author: olivier.soppera@uha.fr
}

Keywords : Nanocomposite, Carbone Nanotubes, Laser treatment, Near-Infrared, Conductive polymer

\begin{abstract}
Near-infrared (NIR) laser annealing is used to write conductive patterns at the surface of

Polypropylene/Multi-walled carbon nanotube nanocomposite (PP/MWCNT) plates. Before irradiation, the surface of the nancomposite is not conductive due to the partial alignment of the MWCNT which occurs during injection molding. We observe a significant increase of the surface sheet resistance using NIR laser irradiation, which we explain by a randomization of the orientation of MWCNTs in the PP matrix melt by NIR laser irradiation. After only $5 \mathrm{~s}$ of irradiation, the sheet resistance of PP/MWCNT, annealed with a laser at a power density of $7 \mathrm{~W} / \mathrm{cm}^{2}$, decreases by more than 4 decades from $\sim 100 \mathrm{M} \Omega / \mathrm{sq}$ to $\sim 1 \mathrm{k} \Omega /$ sq. Polarized Raman, TEM, and SEM are used to investigate the changes in the sheet resistance and confirm the physico-chemical processes involved. This allows directwriting of conductive patterns using NIR laser at the surface of nanocomposite polymer substrates, with the advantages of a fast, easy, and low-energy consumption process.
\end{abstract}




\section{Introduction}

The introduction of nanofillers in polymer matrices is a privileged approach to introduce specific functions to host matrices ${ }^{1-2}$. Among the possible nanoobjects, carbon nanotubes (CNTs) are today widely used to improve the mechanical ${ }^{3-5}$, thermal ${ }^{6-9}$, or/and electrical properties of polymers ${ }^{10-15}$. Carbon nanotubes, especially multi-walled carbon nanotubes (MWCNTs), can be obtained in large quantities by well-established processes. MWCNTs can be introduced up to a few weight percent $\mathrm{wt} \%$, which is sufficient to significantly modify the properties of host matrices. One of the desired effects is to obtain conductive polymers that may be of interest for applications such as 3D printed electronics ${ }^{16-17}$, electrostatic discharge materials, and electromagnetic interference shielding materials. $^{18-22}$

Many methods to produce such nanocomposite materials can be listed. Among them, melt blending ${ }^{23-}$ ${ }^{24}$ offers a simple way of dispersing nanoparticles in a polymer matrix and it is now widely used in industries to manufacture polymer parts ${ }^{25-28}$. A weight percentage higher than the percolation threshold can be used to obtain electrically conductive materials. ${ }^{29-31}$ For example, Seo et al ${ }^{32,33}$ stated that once the percolation threshold is exceeded ("between 1 and $2 \mathrm{wt} \%$ ") the volume resistivity drops from $10^{9}$ $\Omega . c m$ to reach a value of $10^{3} \Omega . \mathrm{cm}$.

Regarding the surface conductivity of nanocomposites, it was reported that the melt blending processes may induce a forced alignment effect ${ }^{34}$. This alignment does not result in a wellinterconnected network; therefore, surface electrical conductivity is very low, even when the percolation threshold is exceeded in the bulk (typically in the range of $10^{7}-10^{8} \Omega / \mathrm{Sq}$ ). Surface electrical conductivity can be enhanced by thermal annealing. When the treatment temperature surpasses the melting temperature of the polymer, entropic effects break the alignment of CNTs, creating connections between the nanotubes that improve the conductivity of the material.

However, thermal curing suffers from certain limitations. First of all, it requires that the entire part obtained after injection is placed in a furnace. Secondly, the process is long, energy-consuming, and for complex 3D objects, annealing above the melting temperature can damage the shape of the object. 
Finally, the whole surface is treated homogeneously, which prevents applications for which a local effect would be sought, for example, to create conductive circuits on an insulating surface.

In this paper, we demonstrate that we can enhance significantly the surface electrical conductivity of polypropylene/Multi-walled carbon nanotube nanocomposite plates by near-infrared (NIR) laser treatment and use it to generate conductive patterns at the surface of the nanocomposite. The interest of such a treatment lies in a short curing time (a few seconds) while keeping the same efficiency as a global heat treatment in terms of surface conduction enhancement ${ }^{35-38}$. Above all, the laser treatment can be localized in space, allowing the direct writing of conductive patterns. A continuous wave infrared laser was used, due to the strong absorption of CNTs for such wavelengths ( $808 \mathrm{~nm})$, as well as the availability of high-power commercial lasers in this wavelength range.

In this article, we demonstrate the effect of the laser on the surface electrical conductivity, which we compare to classical heat treatments. Then, a study of the physico-chemical phenomenon causing the decrease in sheet resistance at the nanocomposite surface was carried out, mainly by combining polarized Raman and TEM results. These new results confirm results reported by other teams on the possibility to modify the surface properties of PP/MWCNTs nanocomposites by laser irradiation. ${ }^{39,40}$ These results were also compared polypropylene (PP), filled with carbon black (CB). Finally, we illustrate the interest of this approach by showing a basic circuit written by the NIR laser at the surface of the PP nanocomposite plate allowing the lightening of a LED without any post-treatment after laser writing.

\section{Experimental section}

\section{Materials}

A MWCNT-Polypropylene (PP) masterbatch has been produced as described in ${ }^{41}$. Average diameter and average length are, respectively, $9.5 \mathrm{~nm}$ and $1.5 \mu \mathrm{m}$, as measured by TEM. The surface area is 250-300 $\mathrm{m}^{2} / \mathrm{g}$, as determined from BET surface area analysis. This masterbatch was then diluted in a TotalEnergies commercial grade (PPC12642, a grade characterized by a melt flow index MFI of 70 $\mathrm{g} / 10 \mathrm{~min}$.$) .$ 
Plates with a dimension of $60 \times 60 \times 1 \mathrm{~mm}^{3}$ (length $\mathrm{x}$ width $\mathrm{x}$ thickness) were prepared by injection molding in a Dr Boy machine (Imposed temperature profile, from the hopper to the injection die: 200, $220,230,250$ and $250^{\circ} \mathrm{C}$, injection speed $=10 \mathrm{~mm} / \mathrm{s}$, injection pressure $=43$ bars, injection time $=3.92 \mathrm{~s}$, mold temperature $\left.=70^{\circ} \mathrm{C}\right)$. Pure PP plates $(\mathrm{PPC} 12642)$ and PP doped with $4 \mathrm{wt} \%$ of MWCNT were used in this study. Both concentrations are above the percolation threshold, ${ }^{42}$ but the surface electrical conductivity is low due to the injection molding process $(100 \mathrm{M} \Omega / \mathrm{sq})$. Polypropylene/carbon black (PP/CB) nanocomposites were prepared by the same process, with $\mathrm{CB}$ nanoparticles. The CB content is $4 \mathrm{wt} \%$.

\section{Laser treatment}

A Coherent-Compact SE Series continuous Near-infrared NIR laser was used for surface treatment of the nanocomposite plates. The nominal power is $50 \mathrm{~W}$. The wavelength is $808 \mathrm{~nm}$, with a typical circular spot diameter of $0.8 \mathrm{~cm}$, with a homogeneous power density produced by a collimating lens at the end of the fiber.

\section{Characterization}

\section{Electrical measurements}

The sheet resistance $R_{S}$ was the chosen property to evaluate the electrical conductivity at the surface of the nanocomposite. Electrodes were deposited by sputtering of $10 \mathrm{~nm}$ of gold (Cressington Sputter Coater 108auto) with a shadow mask to define square areas. The probe area was a rectangle of $2 \mathrm{mmx} 4 \mathrm{~mm}$. A digital multimeter, Keithly's Series 2636B, was used to monitor the I-V curves. The sheet resistance $R_{\text {sheet }}$ was calculated using the slope of the I-V curve $\left(R_{\text {measured }}\right)$ and the following equation: $R_{\text {sheet }}=2 \times R_{\text {measured }}$.

\section{Polarized Raman spectroscopy}

Polarized Raman spectra of the nanocomposite were recorded using a HORIBA - Model Labram 300 spectrophotometer. The polarized laser $(532 \mathrm{~nm})$ was focused at the surface of the nanocomposite with an $\mathrm{x} 100$ objective (beam diameter of the laser spot about $2 \mu \mathrm{m}$ ). 10 spectra were acquired for each 
sample, and an average spectrum, with standard error and baseline subtraction, was calculated. The spectra were then normalized with the peak at $2885 \mathrm{~cm}^{-1}$ of the PP.

\section{Electron microscopy}

For transmission electronic microscopy (TEM) thin sections (< $100 \mathrm{~nm}$ ) were prepared by ultramicrotomy. These sections were made in three directions: parallel to the injection direction $0^{\circ}$, perpendicular to the injection direction $90^{\circ}$, and at an intermediate angle of $45^{\circ}$ between these two preferred directions. An ARM200F JEOL High-Resolution transmission electron microscope (TEM) was used.

Scanning electron microscopy (SEM) was used to observe the morphological change occurring before and after laser treatment, especially at the surface of the nanocomposite sample (JEOL JSM-7900F LV).

\section{Results and discussion}

This first section is aimed at describing the effect of the laser on the surface conductivity of the material. A parametric study of the irradiation conditions and their impact on the electrical properties of the nanocomposite is proposed. Figure 1.a displays a photograph of the laser head and sample during irradiation. Figure 1.b shows a scheme of the laser procedure and pictures of the samples before and after laser treatment. On both samples, gold electrodes were deposited with an identical pattern throughout the study, which allows us to compare the effect of different parameters.

In this first example, an irradiation time of 5 seconds and a power density of $7 \mathrm{~W} / \mathrm{cm}^{2}$ were used. A change in the surface morphology of the sample after laser treatment was observed in the SEM image shown in Figure 1.c. The insulated area displayed a smoother surface compared to the non-insulated site, proving a melting effect caused by the laser treatment. On a macroscopic scale, the same irradiation conditions caused no noticeable deformation of the nanocomposite plate. This preservation of the integrity of the material is a crucial point sought in this study.

The I-V curves are shown in Figure 1.d. It is verified that the conduction is very low on the sample surface before laser treatment, even though the concentration of MWCNT exceeds the threshold concentration. This result is in line with the envisaged MWCNT alignment process. This point will 
also be confirmed by Raman spectroscopy and microscopy analyses afterward. The effect of the laser treatment is clear. After laser treatment, the I-V curve of the insulated zone presents a linear behavior with a significant curvature slope compared to the slope value measured before the laser treatment. These initial results clearly show the effectiveness of laser treatment in increasing the electrical conductivity of the material. The sheet resistance values given in Figure 1.e were calculated using the model shown in the experimental part. The laser treatment drops the surface sheet resistance by more than 4 orders of magnitude from $8.4 \times 10^{7} \Omega / \mathrm{Sq}$ to $1.8 \times 10^{3} \Omega / \mathrm{Sq}$. Besides, we did not notice any difference of conductivity parallel and perpendicular to the direction of injection.

a)

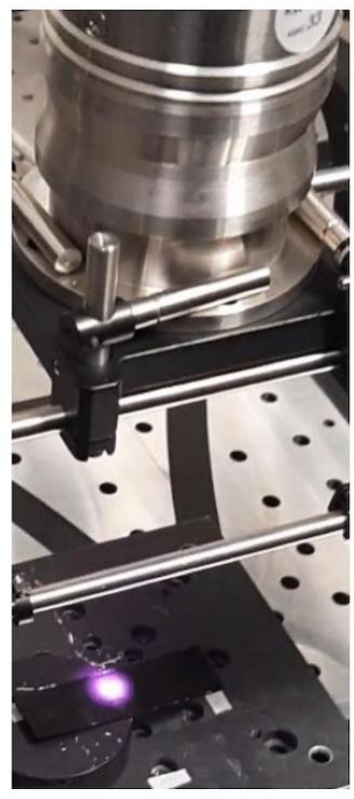

b)
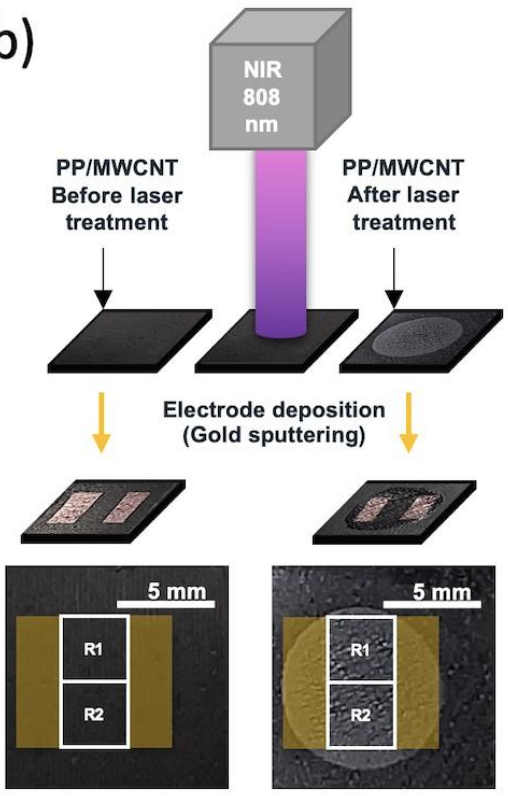

c)
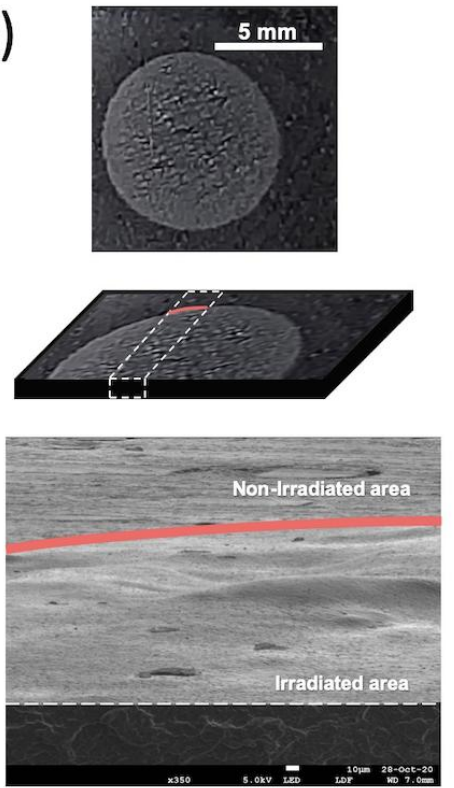

d)

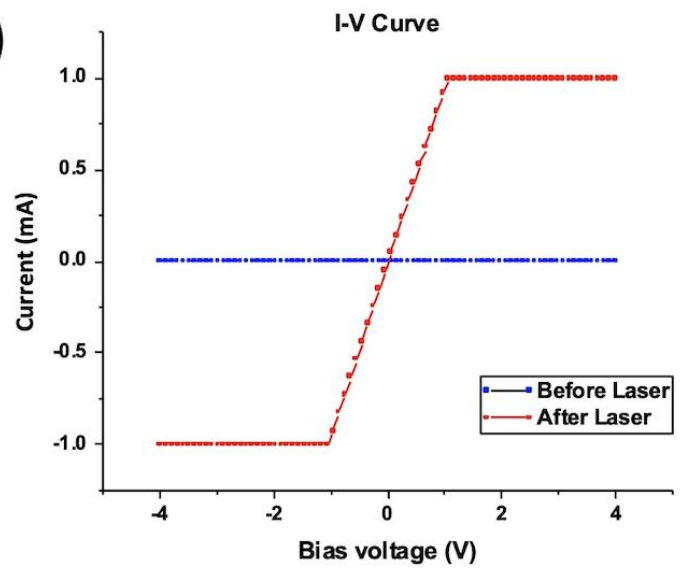

e)

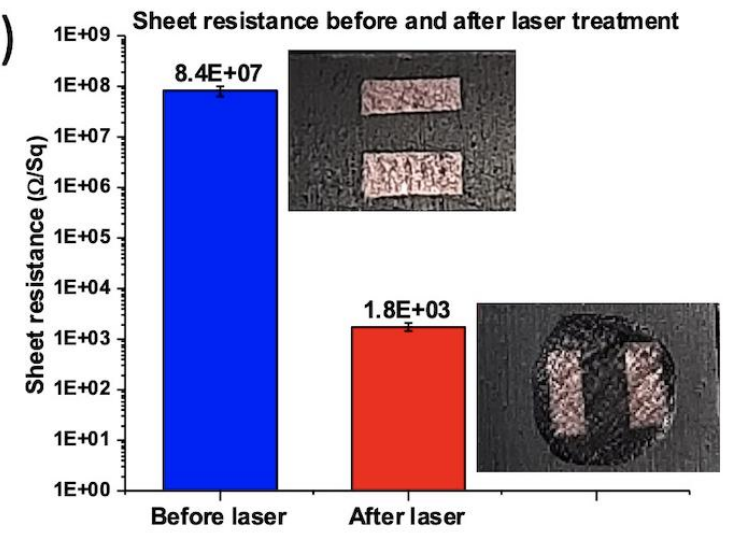

Figure 1. a) Photograph of the sample during NIR laser treatment. b) Schematic of the laser process and pictures of the samples before and after laser treatment (irradiation time of 5 seconds and a power 
density of $7 \mathrm{~W} / \mathrm{cm}^{2}$ ), with gold electrodes. c) SEM image of the sample after laser irradiation (before electrode deposition). d) I-V curves measured before and after laser treatment. e) Sheet resistance measured before, after laser treatment with photographs of the samples.

The effect of laser on the surface conductivity is expected to be very strongly dependent on the irradiation conditions. Figure 2.a shows the evolution of the sheet resistance as a function of the laser energy dose used to irradiate the sample. This energy dose was defined as the multiplication of the power density by the irradiation time. Here, we compared the effect of the dose on the sheet resistance for constant irradiation time and constant power density.

a)

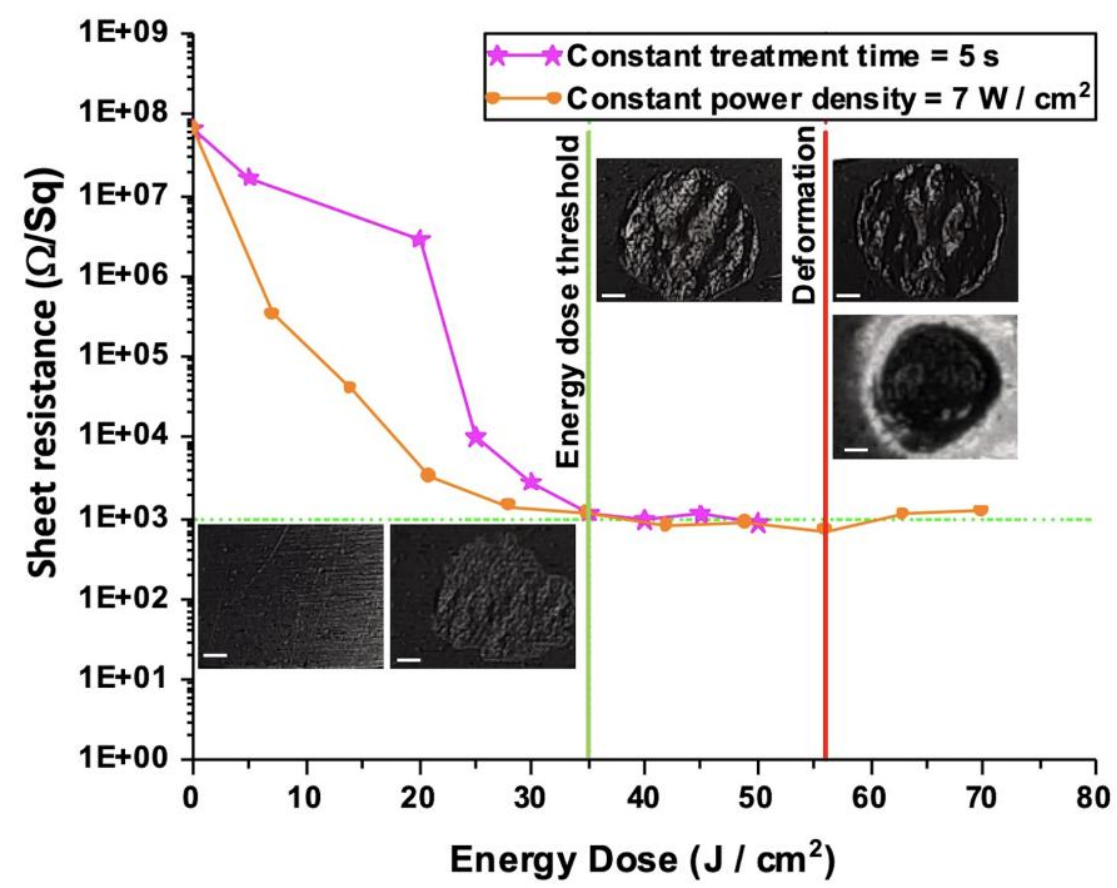

b)
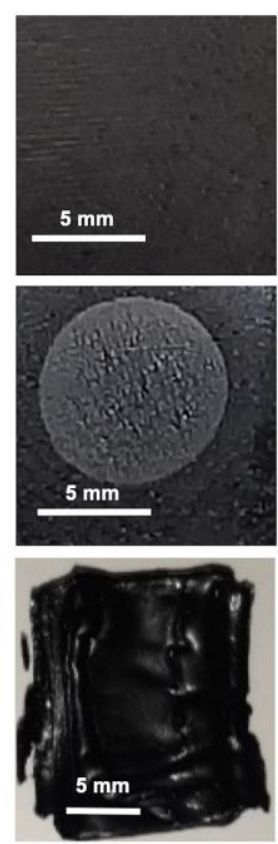

Figure 2. a) Evolution of the sheet resistance versus the light energy dose for a fixed irradiation time $(5 \mathrm{sec})$ with different irradiation power density (pink curve) and a fixed power density $\left(7 \mathrm{~W} / \mathrm{cm}^{2}\right)$ with different irradiation times (orange curve). b) First raw: Picture of samples without laser irradiation; second raw: with laser treatment $\left(5 \mathrm{sec}, 7 \mathrm{~W} / \mathrm{cm}^{2}\right)$; third raw: with thermal treatment ( 3 minutes on a hot plate at $\left.230{ }^{\circ} \mathrm{C}\right)$. Scale bar: $5 \mathrm{~mm}$. 
As expected, the sheet resistance decreases significantly with the increase of laser energy dose in both cases. The minimum value $\left(10^{3} \Omega / \mathrm{Sq}\right)$ was obtained for $35 \mathrm{~J} / \mathrm{cm}^{2}$. Then a plateau is observed up to about $55 \mathrm{~J} / \mathrm{cm}^{2}$. Deformation of the sample surface was observed from this value, which shows that the induced thermal effect is too strong to guarantee the sample's integrity. These conditions were ruled out later on.

There is a significant deviation between experiments conducted at a constant time and a constant power density, for low doses $\left(<35 \mathrm{~J} / \mathrm{cm}^{2}\right)$. This deviation highlights the existence of a threshold power below which the phenomenon is not effectively initiated. This threshold power density can be evaluated at about $4 \mathrm{~W} / \mathrm{cm}^{2}$ (i.e., $20 \mathrm{~J} / \mathrm{cm}^{2}$ for an irradiation duration of $5 \mathrm{~s}$ ). Below this threshold, the heat losses may be such that the melting temperature cannot be reached.

It is interesting to compare these results with those obtained by thermal annealing. To have a point of comparison, the same samples were placed on a hot plate at different temperatures between 50 and $250^{\circ} \mathrm{C}$. An increase in surface conductivity could be qualitatively demonstrated (using an ohmmeter), but no reliable measurement could be made because the samples were deformed entirely under the effect of heat, and it was not possible to rigorously deposit the electrodes by controlling the distance between electrodes (see result at $230^{\circ} \mathrm{C}$, third raw in Figure 2.b). This point demonstrates the decisive advantage of laser treatment over heat treatment.

This second section aims to describe the physico-chemical phenomena explaining the modification of electrical properties induced by the laser. Transmission electron microscopy (TEM) and polarized Raman analyses were performed to describe the evolution of the material upon laser irradiation.

Polarized Raman analysis is very interesting in this context since it allows the detection of the presence of MWCNTs and gives information about their orientations ${ }^{43-53}$. Figure 3.a \& 3.b show the Raman spectra of nanocomposites before and after laser treatment. Polarization directions are usually referred to V (following Ox) and $\mathrm{H}$ (following Oy). Excitation and analysis lights can be polarized. In our case, we consider VV and HH modes, the collection and analysis being done in the same direction. 
The configurations used in this study: VV corresponds to the direction parallel to the injection direction, HH corresponds to the direction perpendicular to the injection direction (Figure 3.c).
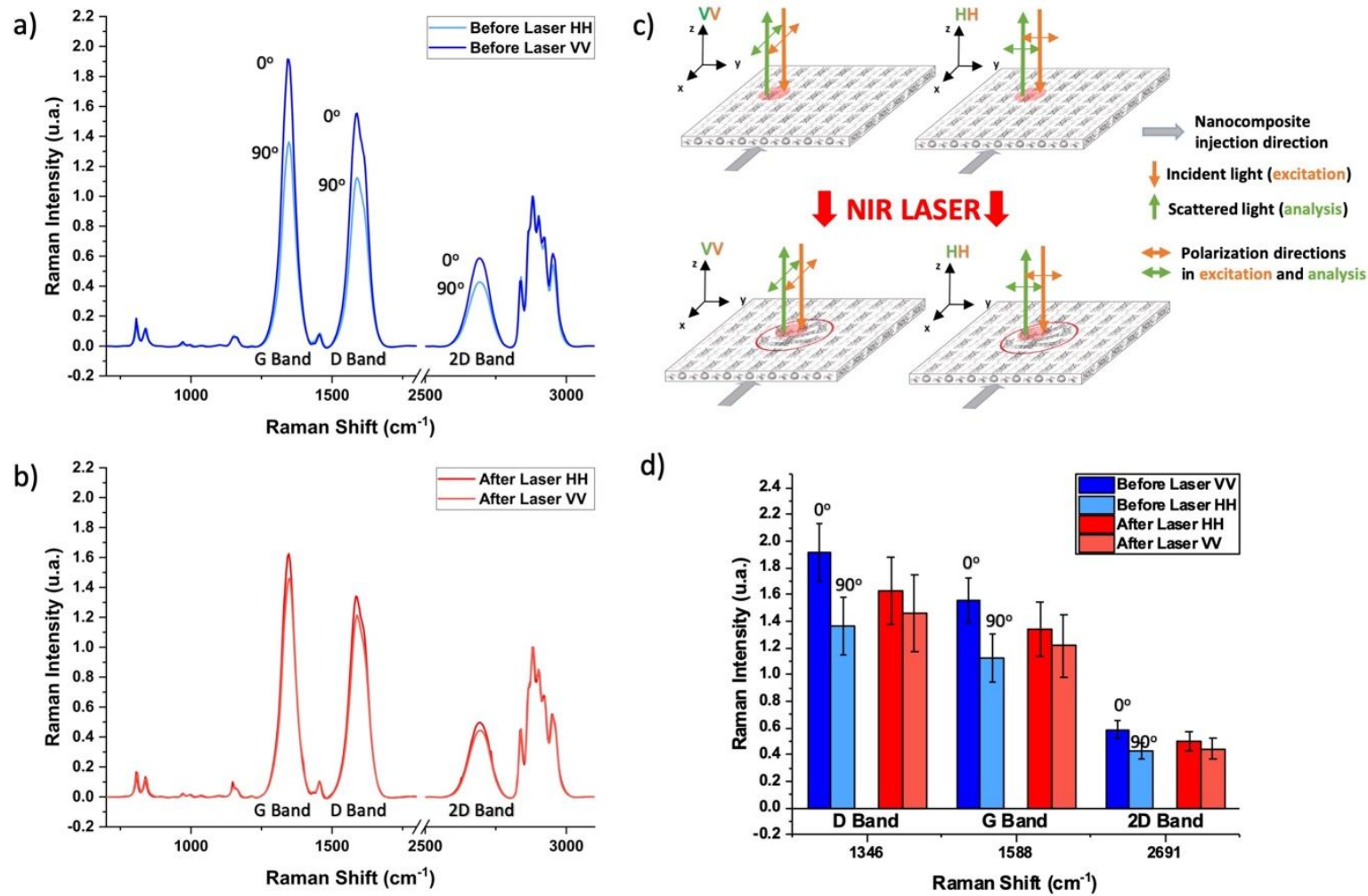

Figure 3: a) Polarized Raman spectra before laser treatment, in two configurations HH \& VV. b) Polarized Raman spectra after laser treatment, in two configurations HH \& VV. c) Schemes representing the $\mathrm{VV}$ and $\mathrm{HH}$ configurations before and after laser treatment as well as the nanotube orientation aligned before treatment then randomized after treatment in the area treated. d) Intensities of Raman MWCNT bands with respect to the laser treatment (irradiation time of 5 seconds and a power density of $7 \mathrm{~W} / \mathrm{cm}^{2}$ ). PP doped with $4 \mathrm{wt} \%$ of MWCNT was used here.

The characteristic bands of PP and CNT are clearly observed in the Raman spectra shown in Figure 3.a \& 3.b. It is possible to distinguish between the characteristic bands of the $\mathrm{PP}(808,841,972,998$, $\left.1151,1168,1220,1425,1458,2704,2850,2885,2940 \mathrm{~cm}^{-1}\right)$ and those of the MWCNT $\left(1347 \mathrm{~cm}^{-1}: \mathrm{D}\right.$ band, $1588 \mathrm{~cm}^{-1}$ : G band, $2692 \mathrm{~cm}^{-1:} 2 \mathrm{D}$ or G' band). Note that the Raman spectra were normalized with respect to the peak at $2885 \mathrm{~cm}^{-1}$ of the PP. We have confirmed the effectiveness of this method 
by measuring the Raman spectra for nanocomposites having different MWCNT concentrations. This method is used in all of the upcoming results.

The Raman spectra before laser irradiation are given in Figure 3.a. The laser was focused at the surface of the sample. Different intensities were observed for the bands of the MWCNTs depending on the direction of polarization (low intensity corresponds to an angle of $90^{\circ}$ and high intensity corresponds to an angle of $0^{\circ}$ with the MWCNTs). As schematized in Figure 3.c, this difference is explained by a preferential orientation of the MWCNTs along the injection direction. This orientation also accounts for the low connection between MWCNTs and thus low surface electrical conductivity shown before.

The Raman spectra after laser treatment are shown in Figure 3.b. The intensity of the bands corresponding to MWCNTs in both polarization directions is almost equivalent and corresponds to a medium intensity compared to $0^{\circ}$ and $90^{\circ}$ before laser irradiation. It can be explained by random angles, as depicted in the Figure 3.c. The analysis of the intensities of the bands is given in Figure 3.d. This Figure shows that the dependence to polarization of the MWCNTs is decreased after laser treatment, which demonstrates that the laser irradiation with a sufficient dose makes it possible to randomize the orientation of the MWCNTs.

For PP, we clearly observe the band at $808 \mathrm{~cm}^{-1}$ which is related to the presence of PP in crystallized form. The relative intensity of this band compared to the band at $840 \mathrm{~cm}^{-1}$ decreases during the laser process, which is in agreement with previously published results, ${ }^{40}$ showing an increase in defects in the crystallized PP phase.

This effect was confirmed by TEM characterization, presented in Figure 4. Thin sections $(<100 \mathrm{~nm})$ were prepared by ultramicrotomy. These sections were made along with three directions: VV (parallel to the injection direction " $0^{\circ}$ "), $\mathrm{HH}$ (perpendicular to the injection direction " $90^{\circ}$ "), and at an intermediate angle of $45^{\circ}$ between these two preferred directions. Samples before and after laser treatment are compared in this Figure. MWCNTs have stronger absorption of the e-beam than the PP matrix, which makes it possible to directly observe them in TEM and define their relative orientation in the sample versus the direction of the e-beam. 

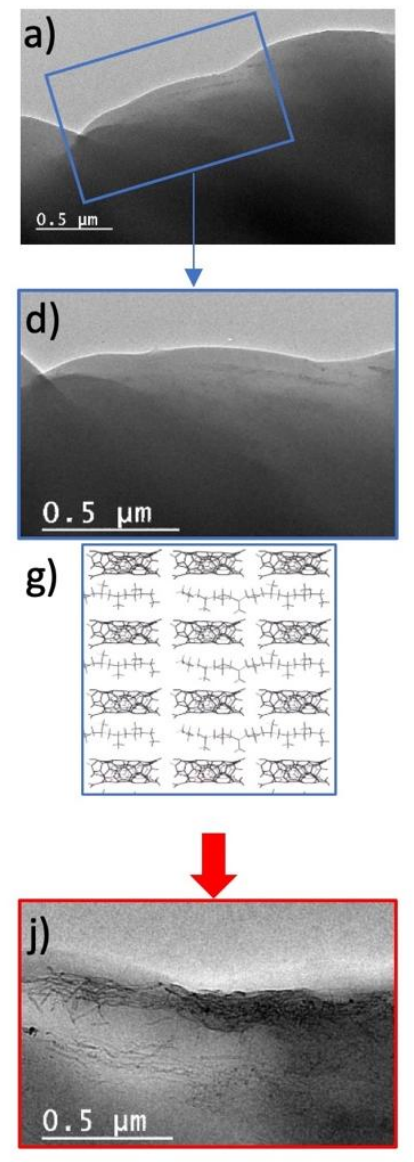
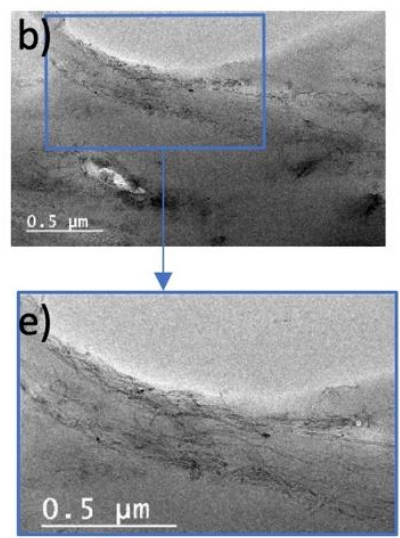

h)
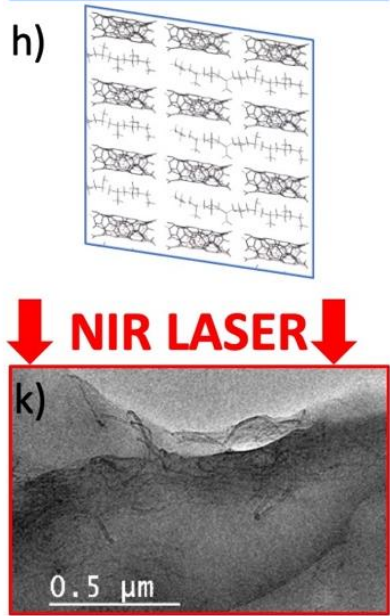
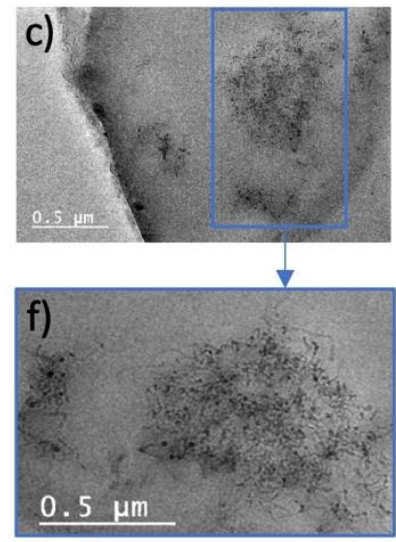

i)

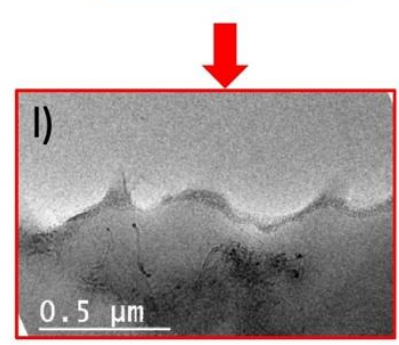

n)
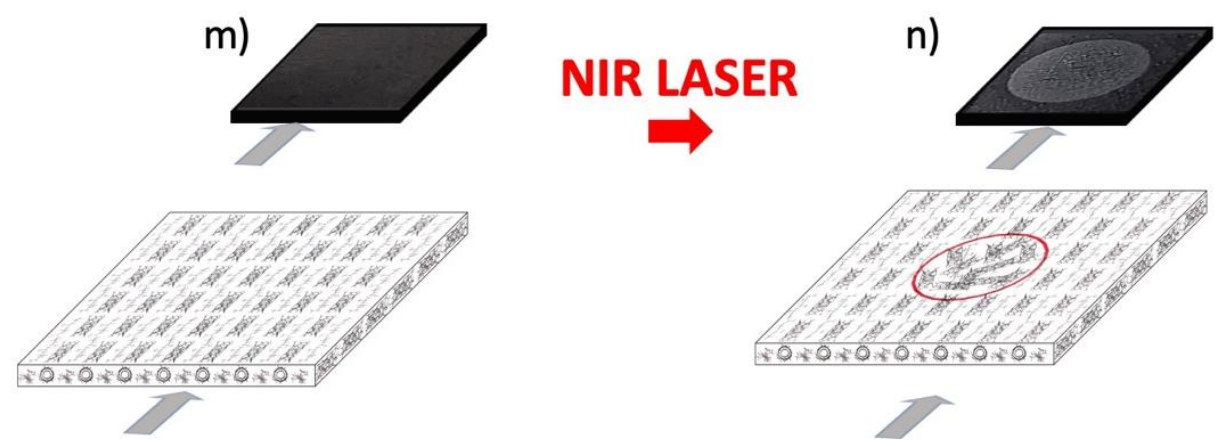

NIR LASER

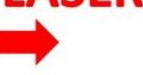

Figure 4. a, b, c) TEM images of the nanocomposite with three different angles of perspective $(0,45$, and $90^{\circ}$ respectively) before laser treatment with zoom in d, e, f). g, h, i) Schemes representing the MWCNTs embedded in the polymer matrix made based on the observation of $d, e, f), j, k, 1)$ TEM images representing three different angles of perspective $\left(0,45\right.$, and $90^{\circ}$ respectively) after laser treatment. $(m, n)$ schemes made based on the results of Polarized Raman and TEM showing the orientation of MWCNTs that changed due to the laser treatment. PP doped with 4 wt $\%$ of MWCNT was used here. Conditions for laser irradiation: irradiation time of 5 seconds and a power density of 7 $\mathrm{W} / \mathrm{cm}^{2}$. 
TEM images, in different observation directions, for samples before laser treatment, are given in Figure 4.a, 4.b \& 4.c, as well as zoom in the zone of interest (Figure 4.d, 4.e \& 4.f). The concentration of MWCNTs in the PP matrix was 4 wt \%. The MWCNTs could not be observed in the sample corresponding to the injection direction $\left(0^{\circ}\right)$ (Figure 4-a $\& \mathbf{d}$ ). If we consider that the injection process aligned the MWCNTs, as shown with Raman, the MWCNTs main axis are, in this projection, perpendicular to the electron beam. In this direction, they can hardly be distinguished from the PP matrix, which can explain why they were not observed by TEM.

At $90^{\circ}$, the TEM images shown in Figure 4.c \& 4.f highlight MWCNTs where the nanotubes appear in the form of black dots. These dots can be seen as MWCNTs oriented with their main axis in the direction of the electron beam, which explains the good contrast. MWCNTs are particularly well visible in some places in the form of agglomerates. It confirms the alignment in the injection direction. The TEM image at $45^{\circ}$ is shown in Figure 4.b \& 4.e. a large number of MWCNTs can be seen, having a higher contrast compared to the TEM image captured at the angle $0^{\circ}$. These observations are consistent with the idea of preferential orientation of the MWCNTs according to the injection direction and are therefore in agreement with the Raman measurements.

After laser treatment, Figure 4.j, 4.k \& 4.l, the anisotropic orientation of the MWCNTs is no longer visible. Indeed, in the three TEM images, MWCNTs are observed in the three cutting directions with almost the same aspects.

These observations by TEM should be considered carefully because one of the difficulties is to ensure the representativeness of the selected sample because it is an observation on a very local scale. On the other hand, coupled with polarized Raman, we can deduce that the initial orientation of MWCNT at the surface of the sample after injection is broken during laser irradiation.

To sum up, a scheme representing the MWCNTs embedded in the polymer matrix before and after laser treatment is presented in Figure 4.m \& 4.n respectively made based on Polarized Raman and TEM results, showing the orientation of MWCNTs that changed due to the laser treatment. The MWCNTs, are aligned due to the injection molding process. Theoretically, the nanocomposite should be conductive regarding to the content of MWCNTs in the PP matrix (4 wt\%) that exceeds the 
percolation threshold, but this was not the case because of the alignment of the MWCNTs. The alignment caused by the manufacturing method prevents the nanotubes from connecting to each other. Therefore, we cannot detect any electrical conductivity even when the percolation threshold is exceeded. The MWCNTs are highly absorbent at the NIR wavelength. Irradiation by the NIR laser makes it possible to locally exceed the melting temperature of the PP, which leads to a random redistribution of the MWCNTs, thus creating the connections between MWCNTs. This physicochemical phenomenon explains the induced electrical conductivity measured at the nanocomposite surface after being insulated with NIR laser.

This hypothesis was confirmed by the last experiment in which we showed that conduction could only be obtained in the presence of MWCNTs, e.g., nanofillers with a very high form factor. We compare hereafter the results obtained with a nanocomposite PP/CB, a PP matrix filled with carbon nanoparticles (carbon black CB).

a)

PP/MWCNT
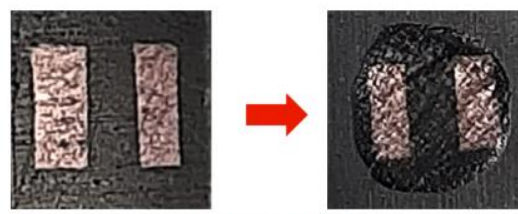

NIR LASER

PP/CB

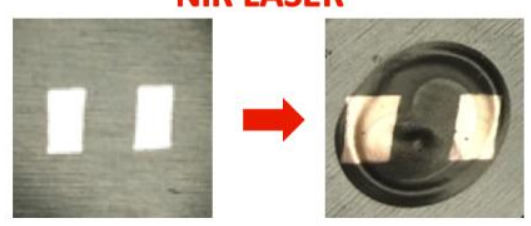

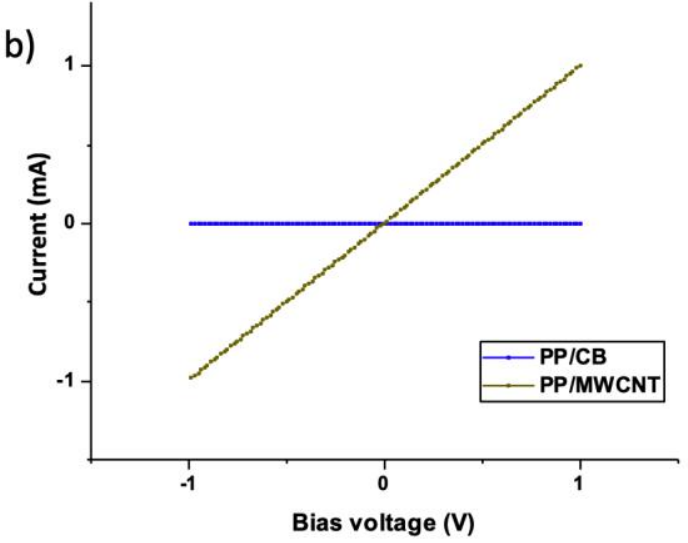

d)

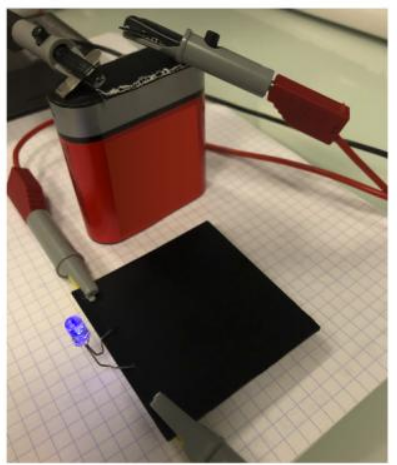

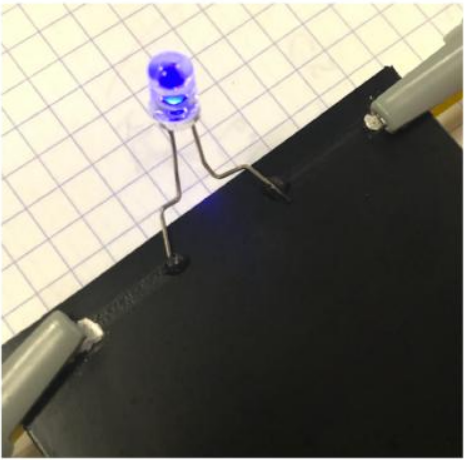

Figure 5. a) Images of PP / MWCNT and PP / CB nanocomposites (4 wt $\%$ for each) before and after laser treatment with gold electrodes. b) I-V curve for PP / CB and PP / MWCNT nanocomposite after laser treatment. Conditions for laser irradiation: irradiation time of 5 seconds and a power density of 7 
$\mathrm{W} / \mathrm{cm}^{2}$. c) Photograph of the experimental setup used for laser direct write of a conductive track. d) Example of conductive track written with $0.75 \mathrm{~W}$ (beam diameter $1.2 \mathrm{~mm}$ ) with a writing speed of 2 $\mathrm{mm} / \mathrm{sec}$. The 2 tracks $(2 \times 1.5 \mathrm{~cm})$ connect a LED that is switched on with a $4.5 \mathrm{~V}$ battery.

The images presented in Figure 5.a show that the interaction between the material and the laser is very effective in the case of $\mathrm{CB}$ nanofiller. The nanocomposite $\mathrm{PP} / \mathrm{CB}$ surface was even more deformed for the same irradiation conditions compared to PP/MWCNT. However, even though this observation proves that the melting point was reached, $\mathrm{PP} / \mathrm{CB}$ had almost no electrical surface conductivity after laser treatment, as shown in Figure 5.b. This result confirms two points about the proposed mechanism. The first point is that the surface electrical conductivity is only obtained with MWCNTs. MWCNTs can generate conduction channels over large distances, and this ability is obtained when contacts occur between them after laser irradiation. The second point is that the surface electrical conductivity cannot be explained by a graphitization of the surface induced by laser treatment. Indeed, this mechanism could be proposed as a possibility considering the high fluence of the laser but it should then be observed not only for MWCNTs but also CB. Moreover, neither the Raman spectra nor the TEM analyses show such a layer. Therefore, we can exclude this mechanism and conclude that the main physico-chemical mechanism is the reorganization by photothermal effect of the MWCNTs, as explained above.

Finally, we illustrate with an example that the laser treatment can be applied on a moving substrate, in order to generate a conductive track of several $\mathrm{cm}$ on the nanocomposite surface (Figure 5.c). In this case, a $1.2 \mathrm{~mm}$ diameter beam is used to irradiate the sample. The sample moves at a speed of 2 $\mathrm{mm} / \mathrm{sec}$ with a translation stage. The power is $0.75 \mathrm{~W}$. With these parameters, each point of the irradiated line receives a power of about $40 \mathrm{~J} / \mathrm{cm}^{2}$, which is of the same order of magnitude as the conditions used in static previously. The written line is visible in Figure 5.d. The resistance of a 1.5 $\mathrm{cm}$ line was measured as $100 \mathrm{k} \Omega$. This value allows, as shown on the photograph, to light an LED powered by a $4.5 \mathrm{~V}$ battery. This example is only an illustration of potential applications of this process useful to write conductive patterns on the surface of a non-conductive material. One can imagine applications in the field of connectors, sensors or antennas for example. 


\section{Conclusion}

We have demonstrated that the surface conductivity of PP/MWCNTs nanocomposites can be locally enhanced by NIR laser irradiation. The impact of the main parameters and the study of the physicochemical phenomena have been carried out. By coupling polarized Raman and TEM studies to surface conductivity measurements, it was possible to show that the absence of conductivity, even for CNT levels above the percolation threshold, can be explained by an alignment of the MWCNTs during the injection process. The strong absorption of MWCNTs in the NIR allows the polymer surface to be heated locally above the melting point. The mobility of the MWCNTs allows a random reorganization of the MWCNTs which creates conduction channels in the material. Thus, a $5 \mathrm{sec}$. irradiation with a power density of $7 \mathrm{~W} / \mathrm{cm}^{2}$ is sufficient to increase the surface conductivity by more than 4 orders of magnitude. These results open the door to the integration of electronic circuits on the surface of plastics by very simple processes. 


\section{References:}

(1) Müller, K.; Bugnicourt, E.; Latorre, M.; Jorda Beneyto, M.; Echegoyen, Y.; Lagaron, J. M.; Miesbauer, O.; Bianchin, A.; Hankin, S.; Bölz, U.; Pérez, G.; Jesdinszki, M.; Lindner, M.; Scheuerer, Z.; Castelló, S.; Schmid, M. Review on the Processing and Properties of Polymer Nanocomposites and Nanocoatings and Their Applications in the Packaging, Automotive and Solar Energy Fields. Nanomaterials 2017, 7, 47, DOI: 10.3390/nano7040074.

(2) Wang, R.; Aakyiir, M.; Qiu, A.; Oh, J.-A.; Adu, P.; Meng, Q.; Ma, J. Surface-tunable, Electrically Conductive and Inexpensive Graphene Platelets and their Hydrophilic Polymer Nanocomposites.

Polymer 2020, 205, 122851, DOI: 10.1016/j.polymer.2020.122851.

(3) Bansal, S.; Kumar, N.; Jindal, P. Effect of MWCNT Composition on the Hardness of PP/MWCNT Composites. Materials Today: Proceedings 2017, 4 (2, Part A), 3867-3871, DOI:

10.1016/j.matpr.2017.02.285.

(4) Kumar, A.; Sharma, K.; Dixit, A. R. A Review on the Mechanical Properties of Polymer Composites Reinforced by Carbon Nanotubes and Graphene. Carbon Letters 2021, 31 (2), 149-165, DOI: $10.1007 / \mathrm{s} 42823-020-00161-\mathrm{x}$.

(5) Wang, B.; Zhang, K.; Zhou, C.; Ren, M.; Gu, Y.; Li, T. Engineering the Mechanical Properties of CNT/PEEK Nanocomposites. RSC Advances 2019, 9 (23), 12836-12845, DOI:

10.1039/C9RA01212E.

(6) Zhou, T. Y.; Tsui, G. C. P.; Liang, J. Z.; Zou, S. Y.; Tang, C. Y.; Mišković-Stanković, V. Thermal Properties and Thermal Stability of PP/MWCNT Composites. Composites Part B: Engineering 2016, 90, 107-114, DOI: 10.1016/j.compositesb.2015.12.013.

(7) Bonnet, P.; Sireude, D.; Garnier, B.; Chauvet, O. Thermal Properties and Percolation in Carbon Nanotube-Polymer Composites. Applied Physics Letters 2007, 91, 201910-201910, DOI:

$10.1063 / 1.2813625$.

(8) Winey, K. I.; Kashiwagi, T.; Mu, M. Improving Electrical Conductivity and Thermal Properties of Polymers by the Addition of Carbon Nanotubes as Fillers. MRS Bulletin 2007, 32 (4), 348-353, DOI: $10.1557 / \mathrm{mrs} 2007.234$.

(9) Jackson, E. M.; Laibinis, P. E.; Collins, W. E.; Ueda, A.; Wingard, C. D.; Penn, B. Development and Thermal Properties of Carbon Nanotube-Polymer Composites. Composites Part B: Engineering 2016, 89, 362-373, DOI: 10.1016/j.compositesb.2015.12.018.

(10) Wang, J.; Kazemi, Y.; Wang, S.; Hamidinejad, M.; Mahmud, M. B.; Pötschke, P.; Park, C. B. Enhancing the Electrical Conductivity of PP/CNT Nanocomposites through Crystal-Induced Volume Exclusion Effect with a Slow Cooling Rate. Composites Part B: Engineering 2020, 183, 107663, DOI: 10.1016/j.compositesb.2019.107663.

(11) Zeng, Y.; Liu, P.; Du, J.; Zhao, L.; Ajayan, P. M.; Cheng, H.-M. Increasing the Electrical Conductivity of Carbon Nanotube/Polymer Composites by Using Weak Nanotube-Polymer iInteractions. Carbon 2010, 48 (12), 3551-3558, DOI: 10.1016/j.carbon.2010.05.053.

(12) Zhou, Y.; Wu, P.; Cheng, Z. Y.; Ingram, J.; Jeelani, S. Improvement in Electrical, Thermal and Mechanical Properties of Epoxy by Filling Carbon Nanotube. eXPRESS Polymer Letters 2008, 2, 4048, DOI: 10.3144/expresspolymlett.2008.6.

(13) Yang, M.; Weng, L.; Zhang, F.; Fan, T.; Zhang, D. Simultaneously Improving the Mechanical and Electrical Properties of Poly(vinyl alcohol) Composites by High-Quality Graphitic Nanoribbons. Scientific Reports 2017, 7, DOI: 10.1038/s41598-017-17365-3.

(14) Park, S. T.; Hwang, J.; Park, G.-S.; Ha, J.-H.; Zhang, M.; Kim, D. E.; Yun, D.-J.; Lee, S.; Lee, S. Modeling the Electrical Resistivity of Polymer Composites with Segregated Structures. Nature Communications 2019, 10, 2537, DOI: 10.1038/s41467-019-10514-4.

(15) Taherian, R.; Ghorbani, M. M. Investigation of the Electrical Properties of Polymer/Carbon Composites Exposed to Joule Heating and Heat Treatment. ECS Journal of Solid State Science and Technology 2017, 6 (6), M3019-M3027, DOI: 10.1149/2.0051706jss.

(16) Ghoshal, S. Polymer/Carbon Nanotubes (CNT) Nanocomposites Processing Using Additive Manufacturing (Three-Dimensional Printing) Technique: An Overview. Fibers 2017, 5 (4), DOI: 10.3390/fib5040040.

(17) Liu, Y.; Xiong, W.; Jiang, L. J.; Zhou, Y. S.; Lu, Y. Precise 3D printing of micro/nanostructures using highly conductive carbon nanotube-acrylate composites, 2016; $\mathrm{p} 973808$. 
(18) Lee, J.-I.; Yang, S.-B.; Jung, H.-T. Carbon Nanotubes-Polypropylene Nanocomposites for Electrostatic Discharge Applications. Macromolecules 2009, 42 (21), 8328-8334, DOI:

10.1021/ma901612w.

(19) Pande, S.; Chaudhary, A.; Patel, D.; Singh, B. P.; Mathur, R. B. Mechanical and Electrical Properties of Multiwall Carbon Nanotube/Polycarbonate Composites for Electrostatic Discharge and Electromagnetic Interference Shielding Applications. RSC Advances 2014, 4, 13839-13849, DOI: 10.1039/C3RA47387B.

(20) Yadav, R.; Tirumali, M.; Wang, X.; Naebe, M.; Kandasubramanian, B. Polymer Composite for Antistatic Application in Aerospace. Defence Technology 2020, 16 (1), 107-118, DOI:

10.1016/j.dt.2019.04.008.

(21) Hosseini, E.; Arjmand, M.; Sundararaj, U.; Karan, K., Filler-Free Conducting Polymers as a New Class of Transparent Electromagnetic Interference Shields. ACS Applied Materials \& Interfaces 2020, 12 (25), 28596-28606.

(22) Zhang, Y.-P.; Zhou, C.-G.; Sun, W.-J.; Wang, T.; Jia, L.; Yan, D.-X.; Zhong, G., Injection Molding of Segregated Carbon Nanotube/Polypropylene Composite with Enhanced Electromagnetic Interference Shielding and Mechanical Performance. Composites Science and Technology 2020, 197, 108253.

(23) Pawar, S. P.; Rzeczkowski, P.; Pötschke, P.; Krause, B.; Bose, S. Does the Processing Method Resulting in Different States of an Interconnected Network of Multiwalled Carbon Nanotubes in Polymeric Blend Nanocomposites Affect EMI Shielding Properties? ACS Omega 2018, 3 (5), 5771 5782, DOI: 10.1021/acsomega.8b00575.

(24) Ke, K.; Wang, Y.; Liu, X.-Q.; Cao, J.; Luo, Y.; Yang, W.; Xie, B.-H.; Yang, M.-B. A

Comparison of Melt and Solution Mixing on the Dispersion of Carbon Nanotubes in a Poly(vinylidene fluoride) Matrix. Composites Part B: Engineering 2012, 43 (3), 1425-1432, DOI:

10.1016/j.compositesb.2011.09.007.

(25) Dönmez, S.; Kentli, A. Influence of Injection Molding Parameters on Electrical Resistivity of Carbon Nanotube Reinforced Polycarbonate. Science and Engineering of Composite Materials 2016, 23 (2), 135-144, DOI: 10.1515/secm-2013-0291.

(26) Mi, D.; Zhao, Z.; Zhu, W. Effects of Three Different Injection-Molding Methods on the Mechanical Properties and Electrical Conductivity of Carbon Nanotube/Polyethylene/Polyamide 6 Nanocomposite. Polymers 2019, 11 (11), 1779, DOI: 10.3390/polym11111779.

(27) Torres-Giner, S.; Chiva-Flor, A.; Feijoo, J. L., Injection-Molded Parts of Polypropylene/Multiwall Carbon Nanotubes Composites with an Electrically Conductive Tridimensional Network. Polymer Composites 2016, 37 (2), 488-496.

(28) Stanciu, N.-V.; Stan, F.; Sandu, I.-L.; Fetecau, C.; Turcanu, A.-M. Thermal, Rheological, Mechanical, and Electrical Properties of Polypropylene/Multi-Walled Carbon Nanotube Nanocomposites. Polymers 2021, 13 (2), DOI: 10.3390/polym13020187.

(29) Zare, Y.; Rhee, K. Y. Simulation of Percolation Threshold, Tunneling Distance, and Conductivity for Carbon Nanotube (CNT)-Reinforced Nanocomposites Assuming Effective CNT Concentration. Polymers 2020, 12 (1), DOI: 10.3390/polym12010114.

(30) Shi, Y.-D.; Lei, M.; Chen, Y.-F.; Zhang, K.; Zeng, J.-B.; Wang, M. Ultralow Percolation Threshold in Poly(l-lactide)/Poly( $\varepsilon$-caprolactone)/Multiwall Carbon Nanotubes Composites with a Segregated Electrically Conductive Network. The Journal of Physical Chemistry C 2017, 121 (5), 3087-3098, DOI: 10.1021/acs.jpcc.6b11351.

(31) Penu, C.; Hu, G.-H.; Fernandez, A.; Marchal, P.; Choplin, L. Rheological and Electrical Percolation Thresholds of Carbon Nanotube/Polymer Nanocomposites. Polymer Engineering \& Science 2012, 52, DOI: 10.1002/pen.23162.

(32) Seo, M.-K.; Lee, J.-R.; Park, S.-J. Crystallization Kinetics and Interfacial Behaviors of Polypropylene Composites Reinforced with Multi-Walled Carbon Nanotubes. Materials Science and Engineering: A 2005, 404, 79-84, DOI: 10.1016/j.msea.2005.05.065.

(33) Seo, M.-K.; Park, S.-J. Electrical Resistivity and Rheological Behaviors of Carbon NanotubesFilled Polypropylene Composites. Chemical Physics Letters - CHEM PHYS LETT 2004, 395, 44-48, DOI: 10.1016/j.cplett.2004.07.047. 
(34) Collet, A.; Serghei, A.; Lhost, O.; Trolez, Y.; Cassagnau, P.; Fulchiron, R. Electrical Conductivity Under Shear Flow of Molten Polyethylene Filled With Carbon Nanotubes: Experimental and Modeling. Polymer Engineering and Science 2021, 61, 1129-1138, DOI: 10.1002/pen.25651 (35) Colucci, G.; Beltrame, C.; Giorcelli, M.; Veca, A.; Badini, C. A Novel Approach to Obtain Conductive Tracks on PP/MWCNT Nanocomposites by Laser Printing. RSC Advances 2016, 6 (34), 28522-28531, DOI: 10.1039/C6RA02726A.

(36) Xiong, W.; Liu, Y.; Jiang, L. J.; Zhou, Y. S.; Li, D.; Jiang, L.; Silvain, J. F.; Lu, Y. LaserDirected Assembly of Aligned Carbon Nanotubes in Three Dimensions for Multifunctional Device Fabrication. Advanced Materials 2016, 9;28(10):2002-9, DOI: 10.1002/adma.201505516. (37) Chang, P. Y.; Bruntz, A.; Vidal, L.; Vetter, P. A.; Roudot, P.; Bua, L.; Ortiz, J.; Zan, H.-W.; Soppera, O. Laser Polymer Tattooing: A Versatile Method for Permanent Marking on Polymer Surfaces. Macromolecular Materials and Engineering 2019, 304, 1900402, DOI:

10.1002/mame.201900402.

(38) Caradonna, A.; Badini, C.; Padovano, E.; Veca, A.; Meo, E.; Pietroluongo, M. Laser Treatments for Improving Electrical Conductivity and Piezoresistive Behavior of Polymer-Carbon Nanofiller Composites. Micromachines 2019, 10, 63, DOI: 10.3390/mi10010063.

(39) Gedvilas, M.; Ratautas, K.; Jagminienè, A.; Stankevičienè, I.; Li Pira, N.; Sinopoli, S.; Kacar, E.; Norkus, E.; Račiukaitis, G., Percolation Effect of a Cu Layer on a MWCNT/PP Nanocomposite Substrate after Laser Direct Structuring and Autocatalytic Plating. RSC Advances 2018, 8 (53), 3030530309.

(40) Ratautas, K.; Gedvilas, M.; Stankevičiene, I.; Jagminienė, A.; Norkus, E.; Li Pira, N.; Sinopoli, S.; Raciukaitis, G., Laser-Induced Selective Metallization of Polypropylene Doped with Multiwall Carbon Nanotubes. Appl Surf Sci 2017, 412, 319-326.

(41) Rousseaux, D.; Lhost, O.; Lodefier, P.; Scandino, E. Patent WO2016/142544A1 2016.

(42) Lecocq, H.; Garois, N.; Lhost, O.; Girard, P.-F.; Cassagnau, P.; Serghei, A.,

Polypropylene/Carbon Nanotubes Composite Materials with Enhanced Electromagnetic Interference

Shielding Performance: Properties and Modeling. Composites Part B: Engineering 2020, 189, 107866.

(43) Guo Liang, G.; Saengchairat, N.; Agarwala, S.; Yeong, W. Y.; Tran, T. Sessile Droplets Containing Carbon Nanotubes: a Study of Evaporation Dynamics and CNT Alignment for Printed Electronics. Nanoscale 2019, 11, DOI: 10.1039/C9NR03261D.

(44) Yang, H.; Fua, B.; Li, D.; Chen, Y.; Mattila, M.; Tian, Y.; Yong, Z.; Yang, C.; Ren, Z.; Bai, J.; Li, Q.; Kauppinen, E.; Lipsanen, H.; Sun, Z. Broadband Laser Polarization Control with Aligned Carbon Nanotubes. Nanoscale 2015, 7, DOI: 10.1039/C5NR01904D.

(45) Liu, J.; Gong, W.; Yao, Y.; Li, Q.; Jiang, J.; Wang, Y.; Zhou, G.; Qu, S.; Lu, W. Strengthening Carbon Nanotube Fibers with Semi-Crystallized Polyvinyl Alcohol and Hot-Stretching. Composites Science and Technology 2018, 164, 290-295, DOI: 10.1016/j.compscitech.2018.06.003.

(46) Qian, H.; Kalinka, G.; Chan, K. L. A.; Kazarian, S. G.; Greenhalgh, E. S.; Bismarck, A.; Shaffer, M. S. P. Mapping Local Microstructure and Mechanical Performance around Carbon Nanotube Grafted Silica Fibres: Methodologies for Hierarchical Composites. Nanoscale 2011, 3 (11), 47594767, DOI: 10.1039/C1NR10497G.

(47) Lee, T.; Park, K. T.; Ku, B.-C.; Kim, H. Carbon Nanotube Fibers with Enhanced Longitudinal Carrier Mobility for High-Performance All-Carbon Thermoelectric Generators. Nanoscale 2019, 11 (36), 16919-16927, DOI: 10.1039/C9NR05757A.

(48) Choi, J.; Jung, Y.; Yang, S. J.; Oh, J. Y.; Oh, J.; Jo, K.; Son, J. G.; Moon, S. E.; Park, C. R.; Kim, H. Flexible and Robust Thermoelectric Generators Based on All-Carbon Nanotube Yarn without Metal Electrodes. ACS Nano 2017, 11 (8), 7608-7614, DOI: 10.1021/acsnano.7b01771.

(49) Arjmand, M.; Mahmoodi, M.; Gelves, G. A.; Park, S.; Sundararaj, U. Electrical and Electromagnetic Interference Shielding Properties of Flow-Induced Oriented Carbon Nanotubes in Polycarbonate. Carbon 2011, 49 (11), 3430-3440, DOI: 10.1016/j.carbon.2011.04.039.

(50) Abbasi, S.; Carreau, P. J.; Derdouri, A. Flow Induced Orientation of Multiwalled Carbon Nanotubes in Polycarbonate Nanocomposites: Rheology, Conductivity and Mechanical properties. Polymer 2010, 51 (4), 922-935, DOI: 10.1016/j.polymer.2009.12.041.

(51) Khan, S. U.; Pothnis, J. R.; Kim, J.-K. Effects of Carbon Nanotube Alignment on Electrical and Mechanical Properties of Epoxy Nanocomposites. Composites Part A: Applied Science and Manufacturing 2013, 49, 26-34, DOI: 10.1016/j.compositesa.2013.01.015. 
(52) Nam, T. H.; Goto, K.; Yamaguchi, Y.; Premalal, E. V. A.; Shimamura, Y.; Inoue, Y.; Arikawa, S.; Yoneyama, S.; Ogihara, S. Improving Mechanical Properties of High Volume Fraction Aligned Multi-Walled Carbon Nanotube/Epoxy Composites by Stretching and Pressing. Composites Part B: Engineering 2016, 85, 15-23, DOI: 10.1016/j.compositesb.2015.09.012.

(53) Beigmoradi, R.; Samimi, A.; Mohebbi-kalhori, D. Engineering of Oriented Carbon Nanotubes in Composite Materials. Beilstein Journal of Nanotechnology 2018, 9, 415-435, DOI:

10.3762/bjnano.9.41. 


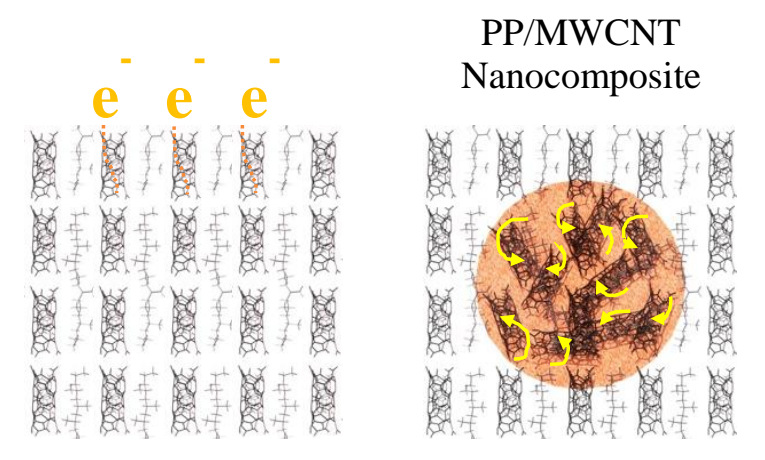

Before laser treatment During laser treatment

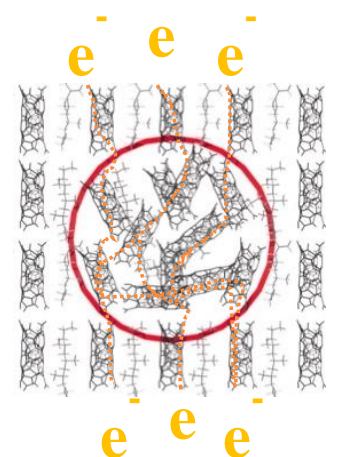

After laser treatment 